\title{
Hungry bone syndrome: still a challenge in the post-operative management of primary hyperparathyroidism: a systematic review of the literature
}

\author{
J E Witteveen ${ }^{1}$, S van Thiel ${ }^{1,2}$, J A Romijn ${ }^{1,3}$ and N A T Hamdy ${ }^{1}$ \\ ${ }^{1}$ Department of Endocrinology and Metabolic Diseases, Leiden University Medical Center, Leiden, The Netherlands, ${ }^{2}$ Department of Internal Medicine, \\ Amphia Medical Center, Breda, The Netherlands and ${ }^{3}$ Department of Medicine, Academic Medical Center, University of Amsterdam, Amsterdam, \\ The Netherlands
}

(Correspondence should be addressed to J E Witteveen; Email: j.e.witteveen@lumc.nl)

\begin{abstract}
Hungry bone syndrome (HBS) refers to the rapid, profound, and prolonged hypocalcaemia associated with hypophosphataemia and hypomagnesaemia, and is exacerbated by suppressed parathyroid hormone (PTH) levels, which follows parathyroidectomy in patients with severe primary hyperparathyroidism (PHPT) and preoperative high bone turnover. It is a relatively uncommon, but serious adverse effect of parathyroidectomy. We conducted a literature search of all available studies reporting a 'hungry bone syndrome' in patients who had a parathyroidectomy for PHPT, to identify patients at risk and address the pitfalls in their management. The severe hypocalcaemia is believed to be due to increased influx of calcium into bone, due to the sudden removal of the effect of high circulating levels of PTH on osteoclastic resorption, leading to a decrease in the activation frequency of new remodelling sites and to a decrease in remodelling space, although there is no good documentation for this. Various risk factors have been suggested for the development of HBS, including older age, weight/volume of the resected parathyroid glands, radiological evidence of bone disease and vitamin D deficiency. The syndrome is reported in 25-90\% of patients with radiological evidence of hyperparathyroid bone disease vs only $0-6 \%$ of patients without skeletal involvement. There is insufficient data-based evidence on the best means to treat, minimise or prevent this severe complication of parathyroidectomy. Treatment is aimed at replenishing the severe calcium deficit by using high doses of calcium supplemented by high doses of active metabolites of vitamin D. Adequate correction of magnesium deficiency and normalisation of bone turnover are required for resolution of the hypocalcaemia which may last for a number of months after successful surgery. Preoperative treatment with bisphosphonates has been suggested to reduce post-operative hypocalcaemia, but there are to date no prospective studies addressing this issue.
\end{abstract}

European Journal of Endocrinology 168 R45-R53

\section{Introduction}

Patients with primary hyperparathyroidism (PHPT) who undergo parathyroidectomy demonstrate a rapid decrease in serum calcium levels after successful removal of one or more hyperactive parathyroid gland(s). This decrease in serum calcium levels is usually mild and maximal 2-4 days post-operatively, and independent of the size of hyperactive glands or pathological diagnosis $(1,2,3,4,5,6,7,8)$. Persistence of hypocalcaemia for more than 4 days after parathyroidectomy may be due to intentional or accidental removal of all parathyroid glands, devascularisation or trauma to residual parathyroid glands, but is also often due to long-term suppression of residual non-pathological parathyroid glands $(1,2,8,9)$.
The term 'hungry bone syndrome' (HBS) has been coined to the profound (serum calcium $<2.1 \mathrm{mmol} / \mathrm{l}$ ) and prolonged (longer than 4th day post-operatively) hypocalcaemia, which follows parathyroidectomy for severe hyperparathyroidism. This is usually associated with skeletal manifestations, reflected by high preoperative indices of bone turnover, osteitis fibrosa cystica and/or 'brown tumours'. The severe hypocalcaemia is believed to be due to the greatly increased skeletal usage of calcium, thought to occur as a result of removal of the effect of high circulating parathyroid hormone (PTH) levels on bone, with immediate arrest of bone resorption in the face of continuing and enhanced bone formation, although there is no good documentation for this.

Literature data on the HBS are scarce despite the still significant prevalence of this clinical problem and 
despite the challenges associated with its management. This has prompted us to perform a systematic review of the literature on this topic. To this effect, we performed a structured literature search in Medline, Embase and the Cochrane Library for studies reporting a 'hungry bone syndrome' in patients who had undergone parathyroidectomy for PHPT.

\section{Methods}

We searched PubMed, EMBASE, Cochrane Library, Web of Science, CINAHL and Science Direct using the following search strategy: ('hypocalcaemia'[ti] OR 'hypocalcemia' [MeSH Terms] OR 'hypocalcemia'[ti] OR Hypocalcemic[ti] OR Hypocalcaemic[ti]) AND (hyperparathyroidism OR parathyroid adenoma OR parathyroid cancer OR 'Parathyroid Neoplasms'[Mesh] OR parathyroidectomy OR hyperparathyroid* OR parathyroidectom* OR 'Hyperparathyroidism/surgery' [Mesh]) AND (postoperative OR post-operative OR Postoperative Complications OR Postoperative Care OR pretreatment OR pretreatment OR prevention OR preventive)) OR ('hungry bone' OR 'hungry bones'). We restricted our search to publications in the 'English language' and on 'Human subjects'. We also checked the references of relevant articles for additional articles. Abstracts of meetings and unpublished results were not included in the study. The last search was performed on January 17, 2012.

\section{Results}

\section{Systematic literature search}

The initial search resulted in a total of 364 articles, 144 of which were excluded based on title and abstract, so that a total of 220 potentially relevant papers were retrieved for full assessment (Fig. 1). Eligibility criteria included articles reporting HBS after surgery for PHPT in adult humans. Exclusion criteria were hypocalcaemia due to any other cause, non-complicated post-operative course, HBS in secondary or tertiary hyperparathyroidism and HBS in children. Comments or Letters to the Editor and articles only displaying a radiological picture were also excluded. One hundred and sixty nine of the 220 publications were excluded based on these exclusion criteria. Consequently, our search strategy ultimately resulted in 51 publications meeting the inclusion criteria of HBS after surgery for PHPT in adult humans.

\section{Pathophysiology of HBS}

Bone remodelling consists of a series of cellular events on the bone surface, the function of which is to remove damaged bone through the process of osteoclastic bone resorption, and replacing it with new bone through the process of osteoblastic bone formation. The process of bone resorption, which lasts about 2 weeks is followed by a reversal phase of 2-3 weeks duration, before new bone is formed, which lasts about 3 months. The remodelling space is the total amount of bone that at any time has been resorbed by osteoclasts but not yet reformed by osteoblasts during the coupled remodelling process because of the delay between resorption and formation. This space depends on the activation frequency of new remodelling sites, which is considerably increased in PHPT, leading to mineral depletion of bone and significantly contributing to the hypercalcaemia of PHPT $(10,11,12,13,14)$.

In those patients with preoperative high rates of bone turnover, successful parathyroidectomy curbs osteoclastic resorption, leading to a decrease in the activation frequency of new remodelling sites and to a decrease in remodelling space leading to a consequent gain in bone mass. This is believed to be the cause of the rapid, profound and sometimes prolonged decrease in

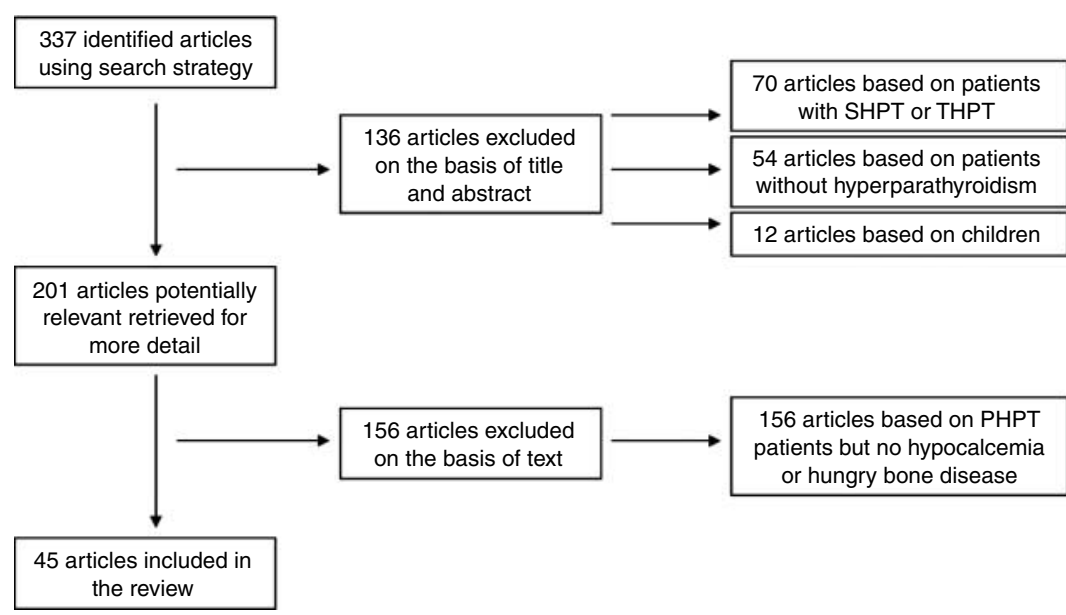

Figure 1 Flowchart of articles included in the systematic review. SHPT, Secondary hyperparathyroidism; THPT, Tertiary hyperparathyroidism; PHPT, Primary hyperparathyroidism. 
serum calcium, phosphate and magnesium levels. The duration of the HBS is defined as the duration of post-operative hypocalcaemia or time required for normalisation of serum calcium following successful parathyroidectomy, which parallels normalisation of bone turnover and may last for up to 9 months, but exceptionally longer in cases of parathyroid carcinoma following radical excision of the tumour. In our experience, the duration of the hypocalcaemia is determined by the height of the increased bone turnover preoperatively as well as by the time required for recovery of normal function of residual non-pathologic parathyroid tissue (N A T Hamdy, 1990-2011, unpublished personal observations).

\section{Clinical manifestations of $\mathrm{HBS}$}

Severe hypocalcaemia (serum calcium concentration $\leq 2.1 \mathrm{mmol} / \mathrm{l}$ ) is associated with neuromuscular irritability, clinically manifested by carpopedal spasms, perioral paresthesiae, tingling extremities, Chvostek sign and Trousseau sign $(15,16,17,18,19,20,21$, $22,23,24,25,26)$. Patients can also develop generalised convulsions, which can lead to pathological fractures $(27,28)$, and ultimately if remaining uncorrected to coma and even death. Congestive heart failure, which is reversible after normalisation of serum calcium concentration, has also been reported $(15,29)$.

\section{Prevalence of HBS after parathyroidectomy}

Data on the prevalence of HBS have been scarce and conflicting after original publications in the 1980s of large case series suggesting that the syndrome develops post-operatively in up to $13 \%$ of patients with PHPT $(1,3,30)$. Recent case series from Asia reported much higher prevalence rates of $24-87 \%(31,32,33,34)$, whereas a case series from Saudi Arabia documented a prevalence rate of only $4 \%$ (35).

\section{Risk factors for the development of HBS}

Age at time of surgery Older age at time of surgery is a risk factor for HBS (1). Brasier \& Nussbaum (1) showed that in a group of 198 patients with PHPT, those who developed HBS were 10 years older than patients with an uncomplicated post-operative course $(61 \pm 3$ vs $51 \pm 1, P<0.05)$.

Laboratory investigations before surgery Patients who developed HBS had higher preoperative levels of serum calcium, and almost two-fold increased levels of PTH and alkaline phosphatase compared with patients who had an uncomplicated post-operative course (Table 1) (1, 4, 36). However, Lee et al. (37) were not able to demonstrate a significant difference in preoperative serum levels of calcium, PTH or alkaline phosphatase between nine patients who developed HBS post-operatively and 14 patients who did not. Serum magnesium and albumin levels were found to be significantly decreased in patients who subsequently developed HBS (Table 1) (1).

There were no available data on the predictive value of preoperative bone markers other than alkaline phosphatase, such as procollagen type 1 aminoterminal propeptide (P1NP, a marker of bone formation) and $\beta$-crosslaps ( $\beta$-CTX, a marker of bone resorption). Depleted vitamin D status (low levels of $25(\mathrm{OH}) \mathrm{D}$ and $\left.1,25(\mathrm{OH})_{2} \mathrm{D}\right)$ has been suggested to be a risk factor for the development of HBS by some, but not all, authors (1, 4, 38).

\section{Radiological bone disease before surgery} Radiological evidence of PHPT-related bone disease has been reported to be an important risk factor for the development of $\operatorname{HBS}(4,16,17,26,27,31,39)$. Fourteen of 18 case reports on HBS indeed report skeletal abnormalities, such as subperiostal erosions, lytic lesions, brown tumours and multiple fractures

Table 1 Preoperative laboratory data in patients with primary hyperparathyroidism who developed HBS following parathyroidectomy compared with those who did not.

\begin{tabular}{llccc}
\hline Laboratory investigation & Authors & $\begin{array}{c}\text { Patients who } \\
\text { developed HBS }\end{array}$ & $\begin{array}{c}\text { Patients who did } \\
\text { not develop HBS }\end{array}$ & $\boldsymbol{P}$ value \\
\hline s-Calcium (mmol/l) & Brasier \& Nussbaum (1) & $3.00 \pm 0.05$ & $2.88 \pm 0.03$ & $<0.05$ \\
& Spiegel et al. (36) & $3.25 \pm 0.05$ & $3.00 \pm 0.03$ & $<0.001$ \\
& Heath et al. (4) & $3.94 \pm 0.38$ & $2.95 \pm 0.15$ & $<0.01$ \\
s-PTH (pmol/l) & Lee et al. (37) & $3.00 \pm 0.1$ & $3.00 \pm 0.08$ & 0.7 \\
& Brasier \& Nussbaum (1) & $10.2 \pm 2.00$ & $5.7 \pm 0.3$ & $<0.05$ \\
s-Alkaline phosphatase (U/l) & Lee et al. (37) & $30.7 \pm 10$ & $32.9 \pm 6$ & 0.2 \\
& Brasier \& Nussbaum (1) & $68 \pm 15$ & $38 \pm 2$ & $<0.05$ \\
s-Magnesium (mEq/l) & Lee et al. (37) & $51 \pm 37$ & $12 \pm 6$ & 0.01 \\
s-Albumin (g/dl) & Brasier \& Nussbaum (1) & $248 \pm 48$ & $169 \pm 31$ & $<0.001$ \\
& Brasier \& Nussbaum (1) & $1.5 \pm 0.1$ & $1.7 \pm 0.04$ & $<0.001$ \\
\hline
\end{tabular}

HBS, hungry bone syndrome; s, serum. 
$(15,16,17,18,19,21,22,23,24,25,26,27,29,40$, $41,42,43,44)$. Osteitis fibrosa cystica was observed in $47-100 \%$ of patients who develop HBS $(31,36)$ and the syndrome was reported in $25-90 \%$ of patients with radiological evidence of PHPT-related bone disease compared with only $0-6 \%$ of patients without skeletal involvement $(4,31,39)$.

Volume and weight of resected pathological parathyroid gland(s) A large study on 198 patients with PHPT demonstrated that the volume and weight of the removed adenomas were significantly greater in patients who developed HBS compared with patients who had an uncomplicated post-operative course $(5 \pm 1$ vs $1 \pm 0.2 \mathrm{~cm}^{3}, P<0.05$ and $4 \pm 1$ vs $2 \pm 0.2 \mathrm{~g}$, $P<0.05$ respectively) (1). Zamboni \& Folse (3) confirmed this finding, by demonstrating that 11 of 16 patients with a single adenoma of $>2 \mathrm{~g}$ developed transient post-operative hypocalcaemia vs only three of 21 patients with a single adenoma of $<1 \mathrm{~g}(P<0.001)$. There are no available data on the relationship between histological characteristics of the resected pathological glands (adenoma vs hyperplasia) and the development of HBS.

\section{Biochemical changes associated with HBS}

A rapid decrease in serum PTH levels to a mean of 1.7 $\pm 0.4 \mathrm{pmol} / \mathrm{l}$ follows successful parathyroidectomy in all cases of PHPT (1). Serum calcium levels drop to $<2.1 \mathrm{mmol} / \mathrm{l}$ within the first $3-4$ days but decrease further after the fourth post-operative day in patients with HBS (1). Serum phosphate levels decrease postoperatively and remain so for the duration of the syndrome $(1,17,27,31,37,38,40,45,46)$. Hypomagnesaemia is frequently encountered (36). Serum alkaline phosphatase levels increase significantly post-operatively and remain elevated sometimes for up to 9 months after surgery $(1,17,27,31,38,39,40$, $42,43,47)$.

Agarwal et al. (31) also reported increased levels of osteocalcin, a marker of bone formation, and decreased urine crosslaps, a marker of bone resorption, in 51 patients 1 week after surgery, with serum osteocalcin levels normalising only 6 months after successful parathyroidectomy (31). In three of 51 patients with extreme osteopenia, bone turnover markers remained elevated for 1 year after successful parathyroidectomy (31).

\section{Radiological changes associated with HBS}

Removal of the excessive circulating levels of PTH shuts off bone-resorptive activity and leads to a rapid increase in bone mineral density. Case reports show an increase in bone mineral density of the lumbar spine of $17 \%$ at
10 weeks, $10 \%$ at 6 months and $27-65 \%$ at 1 year after parathyroidectomy $(7,43,45,47)$ and an increase in bone mineral density of the greater trochanter of $33 \%$ at 6 months and of $35-131 \%$ at 1 year after surgery $(7,47)$.

Bone mineral density increased post-operatively by a remarkable $332 \%$ within 1 year in Indian patients with overt skeletal disease and/or osteitis fibrosa cystica (31). Follow-up radiographs show recovery of subperiosteal resorption and remineralisation of brown tumours, osteolytic lesions and fracture sites $(7,16$, $27,31)$. Skeletal scintigraphy shows an increased radioactive isotope uptake 1 month after parathyroidectomy, known as 'flare phenomenon', which reflects a healing response due to a significant increase in bone formation and consequent mineralisation and high influx of calcium into the skeleton $(16,19)$. A moderately increased uptake can still be seen 8 months after parathyroidectomy (18) and a decrease in the number of lesions and a normalisation of uptake in the remaining lesions 1 year after parathyroidectomy (16).

\section{Management of HBS}

The treatment of HBS is aimed, in the short term, primarily at replenishing the depleted skeletal calcium stores. The first case reports of a HBS, which appeared in the late 1970s, described the difficulties encountered in the management of this severe complication of parathyroidectomy before active metabolites of vitamin $\mathrm{D}$ and their synthetic analogues became available for use in the clinic $(15,22,26,27)$. Persistence of severely decreased serum calcium levels of $\leq 1.3 \mathrm{mmol} / \mathrm{l}$ was thus reported despite treatment with very high doses of calcium, magnesium and cholecalciferol $(15,26,27)$. These management difficulties are, however, still being observed today, despite widespread availability of active vitamin D preparations (23).

The reported amount of calcium supplementation required to treat the severe hypocalcaemia varies between 6 and $12 \mathrm{~g} /$ day $(17,21,23,29,40,43,44)$. Initially, calcium is supplemented intravenously, but treatment with oral preparations should be initiated as early as possible, with concomitant use of adequate doses of active metabolites of vitamin D (calcitriol) or alfacalcidol (2-4 $\mu \mathrm{g} /$ day) $(17,21,22,23,38,42,43$, 44 ), or very occasionally higher doses (unpublished personal observations), and with replenishment of magnesium stores as required. The amount of magnesium required to correct hypomagnesaemia has not always been reported, and supplementation has been variably given intravenously as magnesium chloride or sulphate or orally as magnesium sulphate $(15,17,19$, $22,26,38,43)$. In 11 of 18 cases, serum magnesium level was not mentioned, and in one of 18 cases serum magnesium level was within the normal range $(16,18$, $21,23,24,25,27,29,40,41,42,44)$. 


\section{Treatment options to prevent HBS}

Preoperative treatment with vitamin D Depleted vitamin D status has been postulated to be a risk factor for the development of HBS and it has generally been recommended to supplement vitamin D to normalise $25(\mathrm{OH})$ vitamin D levels, although there are so far no available data to support the premise that this would contribute to the prevention or blunting of $\operatorname{HBS}(1,38)$.

Preoperative treatment with bisphosphonates Two case reports of patients with extensive hyperparathyroid bone disease demonstrated that preoperative treatment with i.v. pamidronate given at a dose of $30 \mathrm{mg}$ for 2 consecutive days or as a single infusion of $60 \mathrm{mg}$, resulted in a preoperative decrease in serum calcium and in a decrease (just $1500 \mathrm{mg}$ calcium orally per day) or no post-operative calcium requirements $(7,48)$. One case report showed that a patient with a history of severe PHPT for longer than 8 years, who was treated with alendronate for 6 years, in addition to receiving a preoperative total dose of $180 \mathrm{mg}$ pamidronate intravenously, did not develop HBS postoperatively (49).

In a retrospective study, Lee et al. (37) also demonstrated that none of six patients who had received bisphosphonates preoperatively (either oral clodronate $400-1600 \mathrm{mg} /$ day or i.v. pamidronate $60 \mathrm{mg} /$ day) developed HBS post-operatively, compared with nine of 17 patients who had not been preoperatively treated with bisphosphonates. There was no significant difference in preoperative mean serum calcium $(3.00 \pm 0.15$ vs $3.01 \pm 0.04 \mathrm{mmol} / \mathrm{l})$, PTH $(34.8 \pm 11$ vs $33.4 \pm 10 \mathrm{pmol} / \mathrm{l})$ or alkaline phosphatase $(224 \pm 50$ vs $174 \pm 60 \mathrm{U} / \mathrm{l})$ levels between groups. A retrospective case series of 46 patients with severe bone disease, who were treated with i.v. zoledronate preoperatively, also reported a low frequency of postoperative HBS of only 4\% (35). Another retrospective case series of six patients with radiological features of osteitis fibrosa cystica, who were preoperatively treated with bisphosphonates (oral alendronate $20-30 \mathrm{mg} /$ day for 4-6 weeks or a single dose of pamidronate $90 \mathrm{mg}$ or ibandronate $150 \mathrm{mg}$ intravenously), reported that none of the patients needed post-operative i.v. calcium supplementation (47).

In contrast, a case report of a patient with severe, prolonged and extensive bone involvement (florid radiological bone changes) has shown that a single dose of $60 \mathrm{mg}$ i.v. pamidronate combined with calcitriol 1-2 $\mu \mathrm{g} /$ day was able to significantly decrease (but not normalise) serum alkaline phosphatase levels (1600 to $420 \mathrm{U} / \mathrm{l}$ ) but was not able to completely prevent HBS (42). Four other case reports also show that treatment of severe hyperparathyroidism with alendronate $(70 \mathrm{mg} /$ week), pamidronate (twice $90 \mathrm{mg}$ or $5 \times 15 \mathrm{mg}$ i.v.) or zoledronate (twice $4 \mathrm{mg}$ i.v.) was unable to completely prevent $\operatorname{HBS}(23,38,43,44)$.
Preoperative treatment with active metabolites of vitamin $D$ Boyle et al. (50) showed that preoperative treatment of severe hyperparathyroidism with $1,25(\mathrm{OH})_{2} \mathrm{D}$ (calcitriol) at a dose of $2 \mu \mathrm{g} /$ day for $1-10$ weeks significantly decreased preoperative alkaline phosphatase levels in three of seven patients with radiological bone cysts, and three other patients required little i.v. calcium supplementation to a total of $<1 \mathrm{~g}$ in the first 12 post-operative days. In contrast, Heath et al. (4) showed that six patients with PHPT and radiological evidence of bone involvement, who were treated preoperatively with $2 \mu \mathrm{g}$ /day of $1,25(\mathrm{OH})_{2} \mathrm{D}$ for 1 week, were as likely to develop hungry bone disease as patients with PHPT and radiological evidence of bone involvement who did not receive active vitamin $\mathrm{D}$ preparations preoperatively (two of six vs one of six respectively).

\section{Discussion}

HBS is a relatively uncommon complication of parathyroidectomy for severe PHPT associated with preoperative high bone turnover. It is characterised by a rapid, profound and persistent hypocalcaemia associated with hypophosphataemia, hypomagnesaemia and is exacerbated by suppressed PTH levels. The duration of the HBS is the time taken to remineralise the skeleton, which is also mirrored by normalisation of bone turnover markers, by healing of radiological features of osteitis fibrosa cystica and brown tumours and by significant gains in bone mass.

A similar but less severe form of the syndrome may also be observed following medical or surgical treatment of hyperthyroidism associated with high bone turnover, in which hypocalcaemia may occur in up to $46 \%$ of patients (51) and may last for up to 12 weeks after initiation of treatment. In contrast to the case with the HBS of severe PHPT, in treated hyperthyroidism, hypocalcaemia is associated with appropriate significant increases in PTH levels $(52,53)$.

Our literature search suggests that the prevalence of HBS has decreased in the Western World over the last two decades, most likely due to the early detection of still asymptomatic PHPT by routine calcium screening before the effects of high circulating levels of PTH on the skeleton, such as high bone turnover, osteoporosis or osteitis fibrosa cystica, become evident $(38,45)$, although exact numbers are missing.

One of the identified risk factors for a post-operative HBS is older age at the time of surgery. With older age being more often associated with vitamin D deficiency, a decrease in renal $1 \alpha$-hydroxylase activity and lower dietary calcium intakes (1), all three factors potentially contribute to a negative calcium balance and clinical bone disease (4). It has indeed been shown that patients with osteitis fibrosa cystica have lower levels of $1,25(\mathrm{OH})_{2} \mathrm{D}$ than expected, which may be 
due to high levels of serum calcium directly inhibiting renal $1 \alpha$-hydroxylase production, or to hypercalcaemiainduced renal impairment with resulting further decreases in $1 \alpha$-hydroxylase activity (7). A testable hypothesis for the development of bone disease, and for the development of HBS, relates to the possibility that low circulating levels of $1,25(\mathrm{OH})_{2} \mathrm{D}$ with resultant decreased fractional absorption of calcium, leads to undermineralisation of the skeleton $(1,4)$. Low levels of $1,25(\mathrm{OH})_{2} \mathrm{D}$ may thus represent a measurable risk factor for the development of HBS, independently of age, although 25(OH)D deficiency has been proposed to be the more significant risk factor (38).

Preoperative serum alkaline phosphatase levels reflect the state of bone turnover and, therefore, the degree of osteoclast activity and bone resorption. It has been suggested that preoperative serum alkaline phosphatase concentrations may predict the degree and duration of hypocalcaemia after successful parathyroidectomy (46). Other risk factors for the development of HBS include evidence of bone disease, such as osteitis fibrosa cystica, subperiostal bone erosions or bone cysts $(4,15,16,17,26,27,31,39)$ and the volume and weight of the removed hyperactive parathyroid gland(s) $(1,3)$.

Treatment of the HBS is aimed in the short term primarily at replenishing the circulating calcium deficit, and in the longer term, at normalising bone turnover and remineralising the skeleton. Doses of calcium and active vitamin D preparations required and duration of treatment is guided by serum calcium and bone turnover marker levels, aiming at normalisation of bone turnover $(3,15,20,26,27,28,45)$, which may sometimes last in excess of 12 months after successful surgery.

In the early stages of HBS, the doses of calcium supplements necessary to increase and maintain serum calcium concentrations within the normal range are too large to be tolerated orally, and i.v. administration is often required $(15,17,21,22,23,29,40)$. When calcium-containing solutions are given intravenously, administration into large veins or via a central venous catheter is recommended to minimise the risk of local irritation or tissue necrosis by accidental extravasation in surrounding tissues. Electrocardiographic monitoring is recommended as dysrhythmias may occur in case of too rapid correction of the hypocalcaemia (54). In prescribing oral calcium preparations, it is important to realise that different calcium preparations contain different amounts of elemental calcium. Of the available oral preparations, calcium carbonate has the highest percentage of elemental calcium (40\%), followed by citrate salts $(20 \%)$. Other calcium preparations are also available (calcium lactate (13\%), calcium gluconate $(9 \%)$ and calcium glubionate $(6.6 \%))$, although they do not contain sufficient elemental calcium per tablet and compliance may be affected by the large number of tablets required to be taken orally to achieve the same calcium level (20). If high doses of magnesium are required for the treatment of hypomagnesaemia, this should only be given intravenously in adequate dilutions of magnesium sulphate, and not intramuscularly or orally. Lower doses of magnesium can be supplemented as magnesium oxide orally or magnesium sulphate intramuscularly Hypocalcaemia does not resolve until the magnesium deficiency has been corrected $(3,15,26$, $27,28,45)$.

Depleted vitamin D status has been postulated to be associated with an increased risk of developing postoperative hypocalcaemia and HBS $(55,56,57,58)$. Preliminary data suggest that preoperative correction of vitamin D deficiency may decrease levels of PTH and bone turnover, without exacerbating hypercalcaemia $(55,58,59)$. Although the effect of preoperative vitamin $\mathrm{D}$ treatment on post-operative hypocalcaemia has not been evaluated by randomised controlled intervention studies in PHPT, it is our experience that a preoperative replete vitamin D status is associated with a decreased likelihood of a severe or prolonged HBS $(1,38)$.

Bisphosphonates are antiresorptive agents, widely used in the management of osteoporosis and bone disorders associated with increased bone turnover, such as Paget's disease of bone or metastatic bone disease. In hyperparathyroid bone disease these agents inhibit osteoclastic bone resorption and decrease activation frequency of remodelling sites, thus resulting in refilling of remodelling space and increasing mineralisation of bone $(38,60,61)$, In this context, preoperative bisphosphonate treatment would have a potential beneficial effect on the severity and duration of HBS by significantly decreasing or normalising bone turnover before surgery is attempted $(38,48)$. In contrast, short term preoperative treatment may exacerbate post-operative hypocalcaemia by just reducing bone resorption, without allowing time for a coupled decrease in bone formation. There are as yet no prospective studies or randomised control trials addressing the use of bisphosphonates in the prevention or limitation of duration of HBS. Data from case reports and small case series on the beneficial effect of preoperative treatment with bisphosphonates on the HBS in patients with hyperparathyroid bone disease $(7,35,37,51)$ or with longstanding severe PHPT (49) are conflicting (23, $27,35,36,37)$. Some cases thus report failure of preoperative bisphosphonates to prevent HBS, although this was believed to be due to short duration of treatment or low dosage used, as serum alkaline phosphatase levels had not normalised before surgery $(23,38,42,43,44)$.

Because low levels of $1,25(\mathrm{OH})_{2} \mathrm{D}$ are a risk factor for the development of post-operative $\operatorname{HBS}(1,4)$, it has also been hypothesised that preoperative 
supplementation of $1,25(\mathrm{OH})_{2} \mathrm{D}$ could shorten symptomatic hypocalcaemia and hospital course (1, 7, 50). Data on preoperative $1,25(\mathrm{OH})_{2} \mathrm{D}$ supplementation in patients undergoing parathyroidectomy for severe PHPT are also conflicting $(4,50)$. A major limitation of studies, addressing the effect of bisphosphonates and/or active vitamin $\mathrm{D}$ preparations on the prevention of HBS, is the lack of patient randomisation.

\section{Conclusion}

HBS is a relatively uncommon but serious complication of parathyroidectomy for PHPT associated with high bone turnover. There are no clear guidelines for the management of the HBS, but treatment is aimed at replenishing the severe calcium deficit and at restoring normal bone turnover with the use of high doses of calcium and active metabolites or analogues of vitamin D. Adequate correction of magnesium deficiency and normalisation of bone turnover are required for resolution of the hypocalcaemia which may last for a number of months after successful surgery. Adequate preoperative treatment with bisphosphonates may reduce the severity and duration of post-operative hypocalcaemia. Further prospective studies are needed to optimise pre- and post-operative treatment strategies in patients with PHPT and skeletal manifestations at high risk for HBS.

\section{Declaration of interest}

The authors declare that there is no conflict of interest that could be perceived as prejudicing the impartiality of the research reported.

\section{Funding}

This research did not receive any specific grant from any funding agency in the public, commercial or not-for-profit sector.

\section{References}

1 Brasier AR \& Nussbaum SR. Hungry bone syndrome: clinical and biochemical predictors of its occurrence after parathyroid surgery. American Journal of Medicine $1988 \mathbf{8 4}$ 654-660. (doi:10.1016/ 0002-9343(88)90100-3)

2 Kaplan EL, Bartlett S, Sugimoto J \& Fredland A. Relation of postoperative hypocalcemia to operative techniques: deleterious effect of excessive use of parathyroid biopsy. Surgery 1982 92 827-834.

3 Zamboni WA \& Folse R. Adenoma weight: a predictor of transient hypocalcemia after parathyroidectomy. American Journal of Surgery 1986152 611-615. (doi:10.1016/0002-9610(86) 90436-8)

4 Heath DA, Van't Hoff W, Barnes AD \& Gray JG. Value of 1- $\alpha$-hydroxy vitamin $\mathrm{D}_{3}$ in treatment of primary hyperparathyroidism before parathyroidectomy. BMJ 1979 1 450-452. (doi:10.1136/bmj.1.6161.450)
5 Chia SH, Weisman RA, Tieu D, Kelly C, Dillmann WH \& Orloff LA. Prospective study of perioperative factors predicting hypocalcemia after thyroid and parathyroid surgery. Archives of Otolaryngology Head \& Neck Surgery 2006132 41-45. (doi:10.1001/archotol. 132.1.41)

6 Westerdahl J, Lindblom P, Valdemarsson S, Tibblin S \& Bergenfelz A. Risk factors for postoperative hypocalcemia after surgery for primary hyperparathyroidism. Archives of Surgery 2000135 142-147. (doi:10.1001/archsurg.135.2.142)

7 Brossard JH, Garon J, Lepage R, Gascon-Barre M \& D'Amour P. Inhibition of $1,25(\mathrm{OH})_{2} \mathrm{D}$ production by hypercalcemia in osteitis fibrosa cystica: influence on parathyroid hormone secretion and hungry bone disease. Bone and Mineral 199323 15-26. (doi:10.1016/S0169-6009(08)80087-6)

8 Norman JG \& Politz DE. Safety of immediate discharge after parathyroidectomy: a prospective study of 3,000 consecutive patients. Endocrine Practice 200713 105-113.

9 Wong WK, Wong NA \& Farndon JR. Early postoperative plasma calcium concentration as a predictor of the need for calcium supplement after parathyroidectomy. British Journal of Surgery 199683 532-534. (doi:10.1002/bjs.1800830433)

10 Parfitt AM. The actions of parathyroid hormone on bone: relation to bone remodeling and turnover, calcium homeostasis, and metabolic bone disease. Part III of IV parts; PTH and osteoblasts, the relationship between bone turnover and bone loss, and the state of the bones in primary hyperparathyroidism. Metabolism 197625 1033-1069. (doi:10.1016/0026-0495(76) 90133-5)

11 Eriksen EF, Mosekilde L \& Melsen F. Trabecular bone remodeling and balance in primary hyperparathyroidism. Bone 19867 213-221. (doi:10.1016/8756-3282(86)90020-7)

12 Charles P, Mosekilde L \& Jensen FT. Primary hyperparathyroidism: evaluated by 47 calcium kinetics, calcium balance and serum bone-Gla-protein. European Journal of Clinical Investigation 1986 16 277-283. (doi:10.1111/j.1365-2362.1986.tb01342.x)

13 Mosekilde L. Primary hyperparathyroidism and the skeleton. Clinical Endocrinology 200869 1-19. (doi:10.1111/j.1365-2265. 2007.03162.x)

14 Ma YL, Cain RL, Halladay DL, Yang X, Zeng Q, Miles RR, Chandrasekhar S, Martin TJ \& Onyia JE. Catabolic effects of continuous human PTH (1-38) in vivo is associated with sustained stimulation of RANKL and inhibition of osteoprotegerin and geneassociated bone formation. Endocrinology 2001142 4047-4054. (doi:10.1210/en.142.9.4047)

15 Falko JM, Bush CA, Tzagournis M \& Thomas FB. Case report. Congestive heart failure complicating the hungry bone syndrome. American Journal of the Medical Sciences 1976271 85-89. (doi:10.1097/00000441-197601000-00012)

16 Ajmi S, Sfar R, Trimeche S, Ben AK \& Nouira M. Scintigraphic findings in hungry bone syndrome following parathyroidectomy. Revista Española de Medicina Nuclear 201029 81-83. (doi:10.1016/j.remn.2009.10.003)

17 Rathi MS, Ajjan R \& Orme SM. A case of parathyroid carcinoma with severe hungry bone syndrome and review of literature. Experimental and Clinical Endocrinology \& Diabetes 2008116 487-490. (doi:10.1055/s-2007-992155)

18 Kuzucu A, Soysal O \& Savli H. Giant mediastinal parathyroid adenoma presenting with a hyperparathyroid crisis and leading to postoperative hungry bone syndrome. European Journal of Surgery $2002 \mathbf{1 6 8}$ 747-749. (doi:10.1080/11024150201 680036)

19 Chen CH, Wang PW, Liu RT, Tung SC, Jean WY, Lu YC, Kao CL \& Chen L. Ectopic parathyroid adenoma with severe hungry bone syndrome: a case report. Changgeng Yi Xue Za Zhi 199619 196-202.

20 Shoback D. Hypoparathyroidism. New England Journal of Medicine 2008359 391-403. (doi:10.1056/NEJMcp0803050)

21 Hussain A, Mahmood H, Geddoa E, Hamish M \& Reda AH. Parathyroid adenoma and hypercalcemia in a patient presenting 
with multiple pathological fractures of the long bones. International Journal of Surgery 20086 465-468. (doi:10.1016/ j.ijsu.2006.05.018)

22 Scott RD, Falconer CW, Fitzpatrick K \& Proter GM. Magnesium depletion and hypocalcaemia after removal of parathyroid carcinoma. Scottish Medical Journal 197621 37-42.

23 Demirci H, Suyani E, Karakoc A, Toruner FB, Yetkin I, Ayvaz G, Taneri F, Cakir N \& Arslan M. A longstanding hungry bone syndrome. Endocrinologist 200717 10-12. (doi:10.1097/01.ten. 0000257439.69010 .39 )

24 Kim KM, Park JB, Bae KS \& Kang SJ. Hungry bone syndrome after parathyroidectomy of a minimally invasive parathyroid carcinoma. Journal of the Korean Surgical Society 201181 344-349. (doi:10.4174/jkss.2011.81.5.344)

25 Silaghi H, Valea A, Ghervan C \& Silaghi AC. Ectopic intrathyroid parathyroid adenoma: diagnostic and therapeutic challenges due to multiple osteolytic lesions. Case report. Medical Ultrasonography 201113 241-244.

26 Jones CT, Sellwood RA \& Evanson JM. Symptomatic hypomagnesaemia after parathyroidectomy. BMJ 19733 391-392. (doi:10.1136/bmj.3.5876.391)

27 Ahuja MM \& Chopra IJ. Coexistent hyperthyroidism and hyperparathyroidism. Metabolism 196817 854-866. (doi:10.1016/ 0026-0495(68)90150-9)

28 Davies DR \& Friedman M. Complications after parathyroidectomy. Fractures from low calcium and magnesium convulsions. Journal of Bone and Joint Surgery. British Volume $1966 \mathbf{4 8}$ $117-126$

29 Varthakavi P, Thayil G, Amin S, Mehtalia SD, Soneji SL \& Joshi VR. Hypocalcaemic congestive heart failure - a postparathyroidectomy complication. Indian Heart Journal $1985 \mathbf{3 7}$ 311-314.

30 Anderberg B, Gillquist J, Larsson L \& Lundstrom B. Complications to subtotal parathyroidectomy. Acta Chirurgica Scandinavica 1981 147 109-113.

31 Agarwal G, Mishra SK, Kar DK, Singh AK, Arya V, Gupta SK \& Mithal A. Recovery pattern of patients with osteitis fibrosa cystica in primary hyperparathyroidism after successful parathyroidectomy. Surgery 2002132 1075-1083. (doi:10.1067/msy.2002. 128484)

32 Gopal RA, Acharya SV, Bandgar T, Menon PS, Dalvi AN \& Shah NS. Clinical profile of primary hyperparathyroidism from western India: a single center experience. Journal of Postgraduate Medicine 201056 79-84. (doi:10.4103/0022-3859.65279)

33 Bhansali A, Masoodi SR, Reddy KS, Behera A, das Radotra B, Mittal BR, Katariya RN \& Dash RJ. Primary hyperparathyroidism in north India: a description of 52 cases. Annals of Saudi Medicine 200525 29-35.

34 Pradeep PV, Jayashree B, Mishra A \& Mishra SK. Systematic review of primary hyperparathyroidism in India: the past, present, and the future trends. International Journal of Endocrinology 20112011 921814. (doi:10.1155/2011/921814)

35 Malabu UH \& Founda MA. Primary hyperparathyroidism in Saudi Arabia: a review of 46 cases. Medical Journal of Malaysia 200762 394-397.

36 Spiegel AM, Marx SJ, Brennan MF, Brown EM, Downs RW Jr, Gardner DG, Attie MF \& Aurbach GD. Parathyroid function after parathyroidectomy: evaluation by measurement of urinary cAMP. Clinical Endocrinology $1981 \quad 15$ 65-73. (doi:10.1111/j.13652265.1981.tb02749.x)

37 Lee IT, Sheu WH, Tu ST, Kuo SW \& Pei D. Bisphosphonate pretreatment attenuates hungry bone syndrome postoperatively in subjects with primary hyperparathyroidism. Journal of Bone and Mineral Metabolism 200624 255-258. (doi:10.1007/s00774005-0680-x)

38 Graal MB \& Wolffenbuttel BH. Consequences of long-term hyperparathyroidism. Netherlands Journal of Medicine 199853 37-42. (doi:10.1016/S0300-2977(98)00010-2)

39 Raef H, Ingemansson S, Sobhi S, Sultan A, Ahmed M \& Chaudhry M. The effect of vitamin D status on the severity of bone disease and on the other features of primary hyperparathyroidism (pHPT) in a vitamin D deficient region. Journal of Endocrinological Investigation 200427 807-812.

40 Laitinen $\mathrm{O}$. Bone, calcium, and hydroxyproline metabolism in hyperparathyroidism and after removal of parathyroid adenoma. Acta Medica Scandinavica 1977202 39-42. (doi:10.1111/j.09546820.1977.tb16779.x)

41 Meydan N, Barutca S, Guney E, Boylu S, Savk O, Culhaci N \& Ayhan M. Brown tumors mimicking bone metastases. Journal of the National Medical Association 200698 950-953.

42 Hisham AN, Aina EN \& Zanariah H. Recognition and management of hungry bone syndrome - a case report. Medical Journal of Malaysia 200055 132-134.

43 Yong TY \& Li JY. Mediastinal parathyroid carcinoma presenting with severe skeletal manifestations. Journal of Bone and Mineral Metabolism 201028 591-594.

44 Corsello SM, Paragliola RM, Locantore P, Ingraudo F, Ricciato MP, Rota CA, Senes P \& Pontecorvi A. Post-surgery severe hypocalcemia in primary hyperparathyroidism preoperatively treated with zoledronic acid. Hormones 20109 338-342.

45 Natsui K, Tanaka K, Suda M, Yasoda A, Shigeno C, Konishi J \& Nakao K. Oxyphil parathyroid adenoma associated with primary hyperparathyroidism and marked post-operative hungry bone syndrome. Internal Medicine 199635 545-549. (doi:10.2169/ internalmedicine.35.545)

46 Vercauteren M, Boeckx E \& Adriaensen H. Parathyroidectomy and postoperative hypocalcemia: a retrospective study. Acta Anaesthesiologica Belgica 198839 59-63.

47 França TC, Griz L, Pinho J. Diniz ET, Andrade LD, Lucena CS, Beserra SR, Asano NM, Duarte AP \& Bandeira F. Bisphosphonates can reduce bone hunger after parathyroidectomy in patients with primary hyperparathyroidism and osteitis fibrosa cystica. Revista Brasileira de Reumatologia 201151 131-137. (doi:10.1590/ S0482-50042011000200003)

48 Kumar A \& Ralston SH. Bisphosphonates prevent the hungry bone syndrome. Nephron 199674 729. (doi:10.1159/000189483)

49 Gurevich Y \& Poretsky L. Possible prevention of hungry bone syndrome following parathyroidectomy by preoperative use of pamidronate. Otolaryngology - Head and Neck Surgery 2008138 403-404. (doi:10.1016/j.otohns.2007.11.014)

50 Boyle IT, Fogelman I, Boyce B, Thomson JE, Beastall GH, McIntosh WB \& McLennan I. $1 \alpha$-Hydroxyvitamin $\mathrm{D}_{3}$ in primary hyperparathyroidism. Clinical Endocrinology $1977 \quad 7$ (Suppl) 215s-222s. (doi:10.1111/j.1365-2265.1977.tb03384.x)

51 See AC \& Soo KC. Hypocalcaemia following thyroidectomy for thyrotoxicosis. British Journal of Surgery $1997 \mathbf{8 4} 95-97$. (doi:10.1002/bjs.1800840136)

52 Mosekilde L, Christensen MS, Melsen F \& Sorensen NS. Effect of antithyroid treatment on calcium-phosphorus metabolism in hyperthyroidism. I: chemical quantities in serum and urine. Acta Endocrinologica 197887 743-750.

53 Grieff $M$. The hungry bone syndrome after medical treatment of thyrotoxicosis. Annals of Internal Medicine 2003139 706-707.

54 Cooper MS \& Gittoes NJ. Diagnosis and management of hypocalcaemia. BMJ 2008336 1298-1302. (doi:10.1136/bmj. 39582.589433.BE)

55 Grey A, Lucas J, Horne A, Gamble G, Davidson IS \& Reid IR. Vitamin D repletion in patients with primary hyperparathyroidism and coexistent vitamin D insufficiency. Journal of Clinical Endocrinology and Metabolism 200590 2122-2126. (doi:10.1210/jc.2004-1772)

56 Stewart ZA, Blackford A, Somervell H, Friedman K, GarrettMayer E, Dackiw AP \& Zeiger MA. 25-Hydroxyvitamin D deficiency is a risk factor for symptoms of postoperative hypocalcemia and secondary hyperparathyroidism after minimally invasive parathyroidectomy. Surgery 2005138 1018-1025. (doi:10.1016/j.surg.2005.09.018)

57 Silverberg SJ. Vitamin D deficiency and primary hyperparathyroidism. Journal of Bone and Mineral Research 200722 (Suppl 2) V100-V104. (doi:10.1359/jbmr.07s202) 
58 Bollerslev J, Marcocci C, Sosa M, Nordenstrom J, Bouillon R \& Mosekilde L. Current evidence for recommendation of surgery, medical treatment and vitamin D repletion in mild primary hyperparathyroidism. European Journal of Endocrinology 2011165 851-864. (doi:10.1530/EJE-11-0589)

59 Rolighed L, Bollerslev J \& Mosekilde L. Vitamin D treatment in primary hyperparathyroidism. Current Drug Safety 20116 100-107. (doi:10.2174/157488611795684613)

60 Adamson BB, Gallacher SJ, Byars J, Ralston SH, Boyle IT \& Boyce BF. Mineralisation defects with pamidronate therapy for Paget's disease. Lancet $1993 \mathbf{3 4 2}$ 1459-1460. (doi:10.1016/ 0140-6736(93)92935-M)
61 Shane E, Jacobs TP, Siris ES, Steinberg SF, Stoddart K, Canfield RE \& Bilezikian JP. Therapy of hypercalcemia due to parathyroid carcinoma with intravenous dichloromethylene diphosphonate. American Journal of Medicine 198272 939-944. (doi:10.1016/ 0002-9343(82)90855-5)

Received 15 June 2012

Revised version received 1 November 2012

Accepted 13 November 2012 